\title{
La República Popular de China y América Latina: impacto del crecimiento económico chino en las exportaciones latinoamericanas
}

\author{
Daniel E. Perrotti
}

RESUMEN

El papel de la República Popular de China en la economía mundial se ha incrementado significativamente en las últimas décadas, convirtiéndola en un socio estratégico en el comercio exterior de gran parte de América Latina. El comercio bilateral entre la región y la nación asiática alcanzó los 120.000 millones de dólares durante el año 2009. En esta investigación se analiza la elasticidad ingreso de las exportaciones de la región a dicho país. Los resultados muestran que, asumiendo un crecimiento real del producto interno bruto (PIB) chino cercano al 7\% anual, el valor (a precios de 2005) de las exportaciones latinoamericanas a China crecería en torno del 10\% promedio anual entre 2014 y 2019. A su vez, un escenario más conservador, en que se contemple un crecimiento promedio anual de la economía china del 4,5\% para igual período, arrojaría un aumento de las exportaciones cercano al $7 \%$ anual.

PALABRAS CLAVE

CLASIFICACIÓN JEL

AUTOR
Comercio internacional, relaciones económicas internacionales, crecimiento económico, exportaciones, estadísticas comerciales, modelos matemáticos, América Latina, China

F10, F17, F60

Daniel E. Perrotti es funcionario del Instituto Latinoamericano y del Caribe de Planificación Económica y Social (Ilpes) de la cepal, y profesor adjunto regular de la Facultad de Ciencias Económicas de la Universidad de Buenos Aires (UBA), Argentina. daniel.perrotti@cepal.org. 


\section{I}

\section{Introducción}

En el presente trabajo se estima la elasticidad ingreso de las exportaciones latinoamericanas a la República Popular de China ${ }^{1}$ para un conjunto de 17 países de la región, donde se toman en cuenta los aspectos dinámicos de las exportaciones, al tiempo que se consideran las variables de control usualmente señaladas en la literatura de comercio internacional.

El autor agradece los comentarios y sugerencias de los señores René Hernández, funcionario de la CEPAL, y de Rhys Jenkins, profesor de la Universidad de East Anglia, Reino Unido, como también las sugerencias de un revisor anónimo.

${ }^{1}$ De aquí en adelante China.
Para el logro de tal cometido, en la sección II se ofrece una breve descripción del impacto que el país asiático genera en la economía mundial, y de su relación con América Latina. Luego, en la tercera sección, se revisa la literatura acorde con los objetivos del presente trabajo, junto con una referencia a los modelos gravitacionales. A continuación, en la sección IV, se presentan las variables consideradas en los modelos, seguidas de los resultados alcanzados por las dos estimaciones realizadas (para las exportaciones en general y según tipo de productos).

Finalmente, en la quinta sección se señalan algunas conclusiones derivadas de los hallazgos del presente trabajo.

\section{II}

\section{El gigante asiático}

Durante las últimas tres décadas, el papel de China en la economía mundial se ha incrementado significativamente. En ese período, el país asiático ha logrado tasas anuales de crecimiento de dos dígitos del producto interno bruto (PIB), lo que le permitió decuplicar su PIB por habitante facilitando la salida de la pobreza a más de 500 millones de personas ${ }^{2}$. Asimismo, China se ha convertido en la segunda economía mundial, medida por el PIB a paridad de poder adquisitivo.

Su preponderancia es tan amplia que abarca no solo la producción, el consumo y el comercio mundial, sino que también la convierte en uno de los actores más importantes del sector financiero global, siendo el principal acreedor de los bonos del tesoro de los Estados Unidos de América. Incluso el país ha impulsado al yuan como moneda alternativa de reserva internacional (Rosales y Kuwayama, 2012).

La magnitud e incidencia del país asiático en el comercio internacional pueden apreciarse de diferentes

\footnotetext{
${ }^{2}$ Sin embargo, el crecimiento y la inclusión social no implicaron mejoras en el coeficiente de Gini, reflejando incrementos en las desigualdades hacia el interior del territorio chino (Rosales y Kuwayama, 2012).
}

maneras. Una de ellas proviene de considerar que, durante 2009, China se convirtió en el mayor exportador mundial de bienes (1.202 mil millones de dólares) ${ }^{3}$, alcanzando a representar el 9,6\% de las exportaciones mundiales. Asimismo, con respecto a las importaciones, su protagonismo no ha sido menor, ubicándose como el segundo mayor importador del mundo detrás de los Estados Unidos de América, y alcanzando en 2009 el 8\% de las importaciones mundiales (Rosales y Kuwayama, 2012).

Además de la dinámica exportadora, otro de los motores económicos (drivers) de la economía china durante las últimas décadas ha provenido del impulso a las inversiones como motor del crecimiento ${ }^{4}$, a tal punto que su economía presenta un coeficiente de consumo a PIB de los más bajos entre las principales economías

\footnotetext{
${ }^{3}$ Las exportaciones chinas por provincia y ciudad de origen se encuentran claramente diferenciadas. Las cuatro provincias principales (Guangdong, Jiangsu, Zhejiang y Shandong), junto con la ciudad de Shanghai, fueron el origen de más del $75 \%$ del total de las exportaciones del país en 2007 (Rosales y Kuwayama, 2012).

${ }^{4}$ La formación bruta de capital fijo (FBCF) representa alrededor del $40 \%$ del producto chino y en 2009 ese porcentaje se elevó debido a los paquetes de estímulo a la inversión en infraestructura. También repuntó el peso del consumo público (Rosales y Kuwayama, 2012).
} 
mundiales. Sin embargo, en los últimos años China ha orientado su política económica en torno del logro de un mayor dinamismo de su consumo interno ${ }^{5}$.

En lo referente a la oferta, la economía china continúa incrementando su producción agrícola, superando incluso a la de los Estados Unidos de América y de la Unión Europea, en tanto que ha elevado su participación en el sector minero. China genera alrededor del $21 \%$ del valor agregado agrícola mundial, y entre sus principales producciones del agro se encuentran el algodón y el arroz (superando, en ambos casos, el 30\% de la producción mundial), y el maíz (con más del $20 \%$ de producción mundial). Por su parte, las producciones de harina y aceite de soja también superan el $20 \%$ a nivel mundial (Rosales y Kuwayama, 2012).

Pero China no es solo sinónimo de sector primario, muy por el contrario, este país constituye uno de los principales generadores de valor agregado manufacturero a nivel mundial, incluidos los sectores con generación de valor en variadas intensidades tecnológicas. También se han visto importantes avances en el sector de servicios, donde el país asiático ha aumentado su eficiencia, sobre todo en los servicios relacionados con el comercio (transporte, infraestructura física, comunicaciones, servicios empresariales y profesionales, incluidos los servicios financieros) (Rosales y Kuwayama, 2012).

El papel de esta economía como consumidor de envergadura a nivel mundial también es sustantivo: China compra el $53 \%$ de las ventas exteriores de granos de soja, el $28 \%$ de aceite de soja y el $23 \%$ de algodón, al tiempo que ocupa el primer lugar como consumidor mundial de carbón, estaño, zinc y cobre. Con relación a estos últimos, su participación en el consumo mundial de minerales y metales rondó, en 2009, el $40 \%$ en plomo, níquel, estaño, zinc, acero primario, cobre refinado y aluminio. Ese mismo año, consumió un 10\% del petróleo crudo a nivel mundial (Rosales y Kuwayama, 2012).

\section{La relación entre China y América Latina}

Para América Latina, China representa un socio estratégico. El comercio bilateral entre ambas ha crecido cuantiosamente a lo largo de la primera década del siglo XXI, alcanzando los 120.000 millones de dólares durante el año 2009. Además del creciente volumen de comercio, algunos de los países de América Latina obtienen ingresos derivados de los derechos sobre las exportaciones, los

\footnotetext{
5 Véase, por ejemplo, "China vows to boost domestic consumption", en: http://www.usatoday.com/story/money/business/2013/04/17/ china-consumption/2089959/.
}

que se constituyeron en un factor importante para el sostenimiento de las cuentas fiscales, la disminución del endeudamiento público y la acumulación de reservas internacionales (Rosales y Kuwayama, 2012).

En el comercio bilateral, la participación de las exportaciones a China como porcentaje del total exportado por la región latinoamericana ha pasado de un promedio del $1,7 \%$ en los años noventa, a $9,4 \%$ en la primera década del siglo XXI (COMTRADE, s/f).

Estos intercambios se han caracterizado por exportaciones de productos primarios y minerales por parte de América Latina (principalmente soja, metales y petróleo), mientras que las importaciones se concentraron en bienes manufacturados provenientes del país asiático. Con relación a este patrón de intercambio, Rosales y Kuwayama (2012) advierten que "es relevante evitar que nuestro creciente comercio con China reproduzca y refuerce un patrón de comercio de tipo centro-periferia, donde China aparecería como un nuevo centro y los países de la región como la nueva periferia”.

La demanda china por los productos primarios latinoamericanos ha emanado de su proceso de industrialización, en el que los metales han ocupado un papel preponderante (Jenkins, 2011). El dinamismo de dicho proceso ha conllevado no solo incrementos en las cantidades demandadas, sino que también ha provocado una importante presión al alza en los precios de los bienes primarios y minerales, lo que se tradujo en una mejora sustancial en los términos de intercambio de muchos países de América Latina.

Según el trabajo de Jenkins (2011), el "efecto China"6 en la demanda mundial ha repercutido sobre todo en los minerales y los metales, debido a que "China ha alcanzado un nivel de ingresos en que el uso de los metales en relación con el PIB tiende a aumentar en forma significativa. Ello ha sido consecuencia del rápido proceso de industrialización del país, que se ha volcado cada vez más a los metales a medida que la producción ha ido variando de bienes intensivos en trabajo (como la confección) a sectores más intensivos en capital (como los productos eléctricos y electrónicos). La demanda de metales también se ha visto impulsada por la construcción y otros proyectos de infraestructura", tal como se detalla en el gráfico $1^{7}$.

\footnotetext{
${ }^{6}$ Efecto entendido según cuánto más alto fue el precio mundial de los productos básicos en 2007 respecto de lo que hubiese sido si la demanda de China hubiera aumentado al mismo ritmo que la del resto del mundo entre 2002 y 2007.

7 En la hipótesis utilizada por Jenkins (2011): "Para calcular la repercusión del crecimiento excepcional de China en los precios de los productos básicos se asume que los otros factores que afectan a
} 


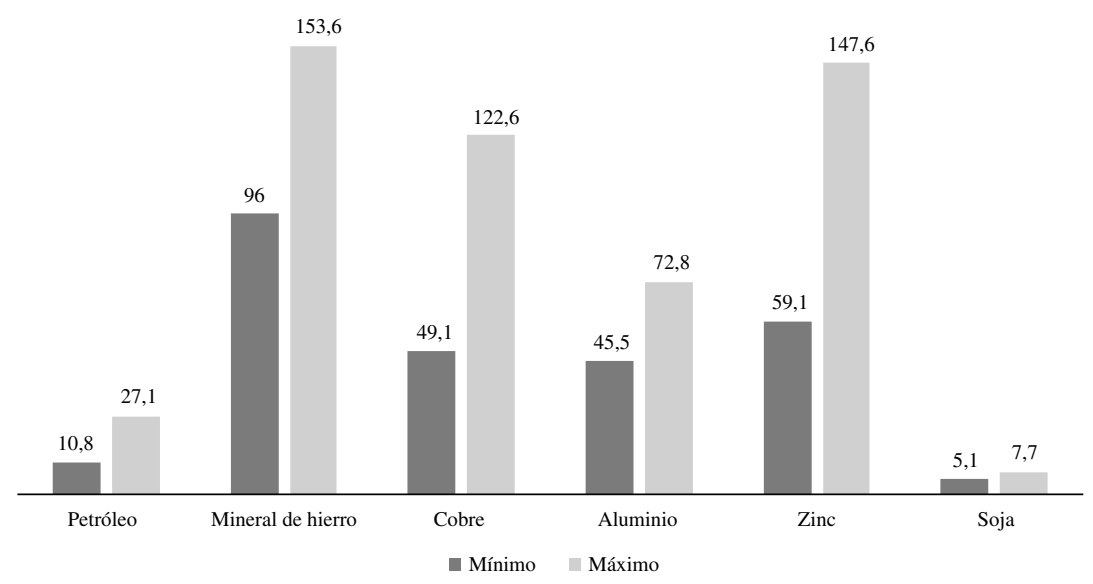

Fuente: elaboración propia sobre la base de R. Jenkins, "El 'efecto China' en los precios de los productos básicos y en el valor de las exportaciones de América Latina”, Revista de la CEPAL, No 103 (LC/G.2487-P), Santiago de Chile, abril de 2011.

a El gráfico muestra cuánto más alto fue el precio mundial de los productos básicos en 2007 respecto de lo que hubiese sido si la demanda de China hubiera aumentado al mismo ritmo que la del resto del mundo entre 2002 y 2007.

Un inconveniente significativo que presenta la canasta exportadora de la región es que compite abiertamente con la de otros países y regiones (como Australia, Canadá, Estados Unidos de América y Nueva Zelandia, y con los países vecinos del país asiático) por el suministro de los productos primarios y minerales a China (particularmente con relación a la minería, la agricultura, la pesca y los productos silvícolas), debido a que China ha diversificado considerablemente sus fuentes de abastecimientos (Rosales y Kuwayama, 2012).

Por otra parte, existe un comportamiento diferenciado hacia el interior de América Latina, especialmente entre Centroamérica, el Caribe y México, por una parte, y América del Sur, por otra. Mientras que esta última, abundante en recursos naturales y agropecuarios, ha sido más beneficiada (con excepción del Paraguay) por el comercio con China y la consecuente elevación en los términos de intercambio derivada del "efecto China" (descrito en Jenkins, 2011), Centroamérica, el Caribe y México no se han visto favorecidos en su comercio con

los precios $[\ldots]$ como las variaciones en las curvas de la oferta, las alteraciones en el tipo de cambio y la especulación, se mantienen constantes. En otras palabras, interesa analizar cuánto más bajos hubieran sido los precios de los productos básicos en 2007 si la participación de China en la demanda mundial se hubiese mantenido en el mismo nivel de 2002, ceteris paribus". el país asiático, en la medida que sus exportaciones son sustitutivas de gran parte de las exportaciones chinas (sobre todo textiles y productos manufacturados), al tiempo que son demandantes netos de recursos naturales (como el petróleo).

En especial, en el trabajo de Jenkins (2011) se clasifica a los países según el impacto del "efecto China", destacándose a los beneficiarios sustanciales, que son las economías de la región exportadoras de minerales (Bolivia (Estado Plurinacional de), Chile y Perú). Luego se encuentran los exportadores de petróleo (Ecuador, México y Venezuela (República Bolivariana de)) y las dos economías más diversificadas de la región (Argentina y Brasil). Finalmente se hallan los países de Centroamérica y el Caribe, que fueron afectados por el alza de los precios internacionales de los productos primarios y minerales, y el caso de México, que se vio en desventaja a causa de la mayor competencia que ha debido enfrentar con las manufacturas chinas en el mercado estadounidense.

En el período reciente, la desaceleración económica de 2008-2009 mostró un incremento en la participación de China como destino de las exportaciones regionales, en detrimento del peso de los Estados Unidos de América. Esto se debió, en parte, a que durante la crisis internacional de 2009, el Gobierno de China impulsó un programa de estímulos contracíclico por 586.000 millones 
de dólares (principalmente a través de inversiones en infraestructura) con el que consiguió evitar los efectos duraderos de la crisis, a tal punto que la economía china creció 9,1\% en 2009, logrando que el comercio exterior se recuperara rápidamente luego del impacto negativo inicial (SELA, 2010).

\section{III}

\section{Revisión de la literatura}

En relación con las circunstancias mencionadas, que dan cuenta de la creciente importancia de China como socio comercial de América Latina, y de su sustantivo papel como referente del comercio mundial, la presente investigación - como se ha señalado en la Introducciónse centra en el análisis de la elasticidad ingreso de las exportaciones latinoamericanas a dicho país, asunto que ha sido estudiado en dos trabajos recientes.

En el primero de ellos, elaborado por Nomura (2013), se analizan tres canales de transmisión de una desaceleración de la economía china hacia el resto del mundo, como son: el exportador, el de los precios de los productos básicos (commodities) y el financiero, para un conjunto de 26 países. El resultado al que llega el estudio considerando los tres canales de transmisión, y al comparar un escenario base con uno de riesgo ${ }^{8}$, es que una caída de un punto porcentual en el PIB de China reduciría el crecimiento económico fuera de ese país en 0,3 puntos porcentuales del PIB. En particular para el caso de América Latina, se constata un impacto incluso mayor de 0,5 punto porcentual por cada punto de disminución en el PIB del gigante asiático.

En el mismo trabajo se señala que la elasticidad de las importaciones chinas sobre el hierro, el petróleo crudo y el cobre ascienden a 4,2; 5,$7 ; \mathrm{y}-0,2$, respectivamente ${ }^{9}$. Sin embargo, estas elasticidades surgen de estimaciones con modelos simples, en que no se consideran efectos dinámicos $u$ otras variables adicionales a las del crecimiento del PIB de China, lo que se suma al hecho del reducido número de países considerados en la publicación bajo el concepto de América Latina (Brasil, Colombia, Chile y México).

\footnotetext{
8 Para las estimaciones de este escenario, en el trabajo de Nomura (2013) se asume una caída en el precio de los metales de entre el $20 \%$ y el $30 \%$, y de entre el $15 \%$ y el $20 \%$ respecto del precio del petróleo para el año 2014, tomando como base el promedio del año 2013.

${ }^{9}$ En el trabajo de Nomura (2013) se señala que la elasticidad negativa del cobre puede ser atribuida al negocio financiero del metal rojo en China.
}

Por su parte, en un documento de la CEPAL (2012) se analiza el impacto de la demanda mundial y de los precios de los principales productos de exportación de América Latina y el Caribe mediante la aplicación de modelos de series de tiempo ARIMA y modelos gravitacionales ${ }^{10}$. En el trabajo se señala que el modelo gravitacional sirvió de base para calcular las elasticidades ingreso de las exportaciones de cada país de la región a los principales destinos, incluida China. A partir de dichas elasticidades se procedió a estimar el volumen de las exportaciones de los diferentes países ${ }^{11}$ en el período 2012-2015, asumiendo determinadas proyecciones para el PIB de las economías de América Latina y el Caribe y de China.

En este estudio, utilizando el modelo gravitacional, se concluye que la elasticidad ingreso de las exportaciones de América Latina y el Caribe hacia China asciende a 2,3, la que se corresponde con el promedio ponderado de las elasticidades que se calculan individualmente para cada país, empleando como ponderadores a las exportaciones según destino. En comparación con otras regiones, y tal como se puede observar en el cuadro 1, China registra, junto con el resto de Asia, la mayor elasticidad ingreso de las exportaciones de América Latina y el Caribe.

\footnotetext{
${ }^{10}$ Las especificaciones que señalan los autores en cuanto a estos modelos son las siguientes: "Al aplicar el modelo ARIMA, se consideraron datos mensuales del período comprendido entre enero de 2006 y junio de 2012 sobre exportaciones en valores corrientes y precios por categorías de productos, y se obtuvo una proyección del volumen exportado de algunos grupos de productos. En el caso del modelo gravitacional, se utilizaron flujos anuales bilaterales de comercio del período 1995 2009 y un conjunto de variables explicativas usuales en este tipo de modelos (PIB, distancia, mediterraneidad, idioma común, existencia de acuerdos de comercio)" (CEPAL, 2012).

11 En el trabajo no se aclara el listado de países incluidos en las estimaciones.
} 
CUADRO 1

Elasticidad ingreso de las exportaciones de América Latina y el Caribe

\begin{tabular}{lc}
\hline País o región & Elasticidad ingreso \\
\hline Estados Unidos de América & 1,7 \\
Europa & 1,9 \\
República Popular de China & 2,3 \\
Resto de Asia & 2,3 \\
\hline
\end{tabular}

Fuente: Comisión Económica para América Latina y el Caribe (CEPAL), Panorama de la Inserción Internacional de América Latina y el Caribe, 2011-2012 (LC/G.2547-P), Santiago de Chile, 2012. Publicación de las Naciones Unidas, N de venta: S.12.II.G.5.

\section{IV}

\section{Estimaciones}

La literatura tradicional sobre los modelos de comercio internacional se basa en los denominados modelos gravitacionales (gravity models), los cuales fueron originalmente introducidos en economía por Tinbergen en el año 1962. La denominación "gravitacionales" proviene de la analogía con la física, específicamente con la Ley de Gravedad de Newton, donde una masa de bienes o factores productivos, en un punto de origen, es atraída por una masa demandante en un punto de destino (Anderson, 2010).

Estos modelos, en sus formas básicas, parten de la presunción teórica de que los flujos de comercio se encuentran relacionados directamente con el tamaño de la economía de los países que comercian, e inversamente con su distancia. En forma genérica, el comercio (" $X$ ”) es función de los "atractores" económicos (" $m$ ", PIB de los países), la distancia (“ $d$ "), y las políticas comerciales, " $p$ " (De Benedictis y Taglioni, 2011):

$$
X=f(m, d, p)
$$

Los modelos gravitacionales se estiman habitualmente de manera transversal, es decir, considerando a un conjunto diverso de países en un momento dado del tiempo. En este sentido, el presente trabajo se ha alejado de dicha tradición en la medida en que el foco de análisis ha tenido en cuenta únicamente el comercio de América Latina con China en un espacio temporal amplio. Asimismo, de manera natural, tampoco se ha considerado la variable distancia, debido a que esta permanece constante en el tiempo.

\section{Variables consideradas}

En los datos tenidos en consideración para las estimaciones se incluye, como se mencionó en la Introducción, a 17 países de América Latina, a saber: Argentina, Bolivia (Estado Plurinacional de), Brasil, Chile, Colombia, Costa Rica, Ecuador, El Salvador, Guatemala, Honduras, México, Nicaragua, Panamá, Paraguay, Perú, Uruguay y Venezuela (República Bolivariana de). En la información temporal, de frecuencia anual, se contempla el período $1990-2013^{12}$.

Para los fines de encontrar la elasticidad ingreso de las exportaciones a China, se ha trabajado con una especificación que tuvo en cuenta las siguientes variables tradicionales en la literatura económica del comercio internacional, que en su forma genérica viene dada por:

$$
\begin{gathered}
x_{i, t}=f\left(y_{t}, \text { tot }_{i, t}, \text { tcr }_{i, t}, r_{i, t}\right) \\
+++\quad+
\end{gathered}
$$

donde:

i) “ $x$ " representa a las exportaciones valoradas a dólares constantes, del país "i" con destino a China en el año " $\mathrm{t}$ ", las que han sido obtenidas de la Base de

\footnotetext{
12 Para las estimaciones se ha restringido el período de la muestra debido a motivos metodológicos que se explican más adelante, y que coinciden con el interés particular del autor del presente artículo de estimar las elasticidades correspondientes al período de gran auge en las relaciones comerciales entre América Latina y China, que se observó en la primera década del siglo XXI, tal como lo señalan Rosales y Kuwayama (2012).
} 
Datos Estadísticos sobre el Comercio de Mercaderías (COMTRADE), y corresponden a la nomenclatura del Sistema Armonizado (HS). Las series han sido deflactadas utilizando los deflactores del comercio exterior (índice base $2005=100$ ) de cada país, obtenidos de CEPALSTAT. En el gráfico 2 se puede observar que la región mostró un crecimiento de las exportaciones a China a razón del 19\% promedio anualizado entre los años 1990 y 2013, destacándose el gran impulso registrado en la primera década del siglo XXI, donde mostró un incremento promedio anualizado del $24 \%$.

ii) " $y$ " corresponde al PIB chino, medido en miles de millones de yuanes a valores constantes, obtenidos de la base de datos de Indicadores del Desarrollo Mundial del Banco Mundial. En el gráfico 3, se observa el crecimiento ininterrumpido que ha tenido esta economía en las últimas décadas, la que se incrementó en torno del 10\% anualizado entre los años 1990 y 2013.

GRÁFICO 2

Exportaciones de América Latina a China, 1990-2013

(En millones de dólares a precios de 2005)

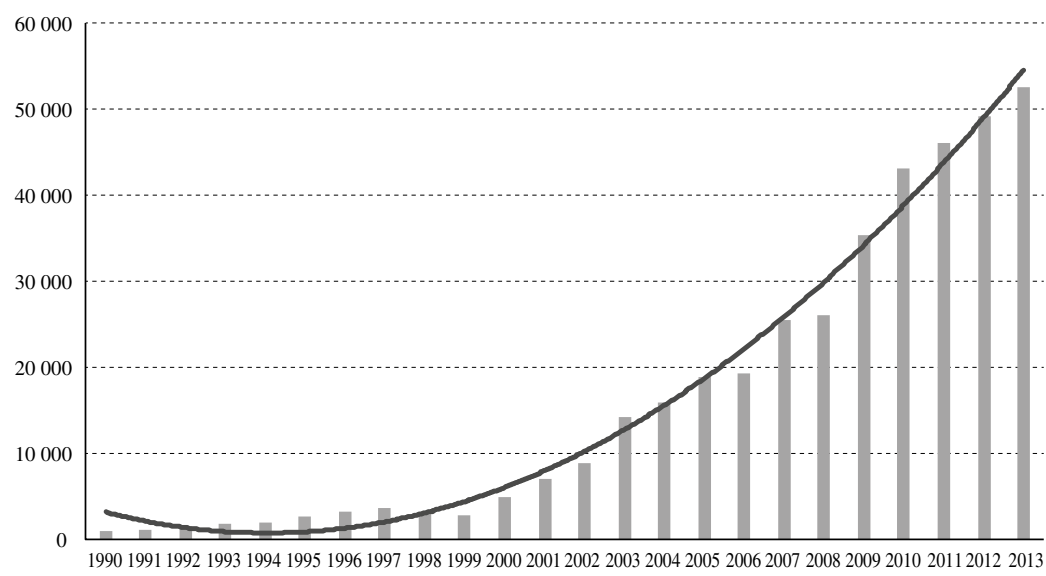

Fuente: elaboración propia a partir de información de la Base de Datos Estadísticos sobre el Comercio de Mercaderías (COMTRADE).

GRÁFICO 3

China: producto interno bruto (PIB), 1990-2013

(En miles de millones de yuanes a precios constantes)

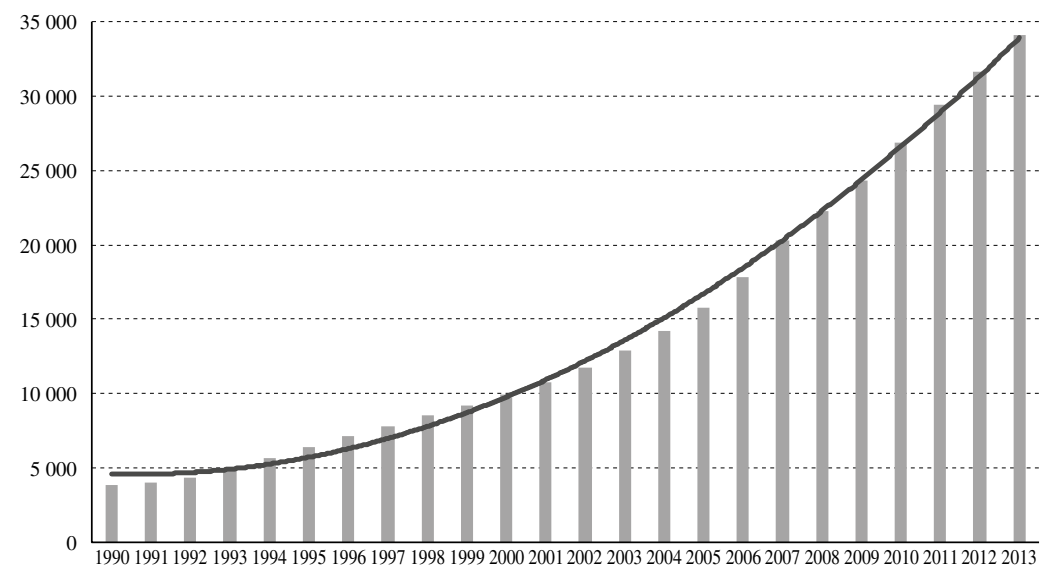

Fuente: elaboración propia sobre la base de datos del Banco Mundial. 
iii) "tot" da cuenta del índice de relación de precios de intercambio y poder de compra de las exportaciones de América Latina, medido sobre la base 2005 = 100, obtenido de CEPALSTAT. En el gráfico 4 se puede apreciar que, considerando el promedio simple de la evolución de los términos de intercambio de todos los países de la muestra, se destaca que desde el año 2003 en adelante ha habido una tendencia de incremento positivo significativo en la evolución de los "tot", con algunos puntos de reversión (pero que hasta el momento no afectaron a la tendencia), como la crisis de 2009 y el último período observado (2012 y 2013), aunque las cifras se mantienen claramente por sobre lo registrado durante la década de 1990. iv) "tcr" constituye el tipo de cambio real bilateral entre China y el país "i”", sobre la base $2005=100$, obtenido a partir de los tipos de cambio nominales bilaterales $\left(\mathrm{tcn}^{\mathrm{i}}\right)$, los que fueron deflactados por los índices de precios al consumidor de los respectivos países (ipci), e indexados por igual indicador de precios referente a China (ipc $\left.{ }^{\text {china }}\right)$, tal como se muestra en la siguiente ecuación:

$$
T C R B_{i, t}=\frac{t c n_{t}^{i} \cdot i p c_{t}^{c h i n a}}{i p c_{t}^{i}}
$$

Las series utilizadas para armar este indicador fueron obtenidas del Banco Mundial y de CEPALSTAT.

GRÁFICO 4

América Latina: términos de intercambio, 1990-2013

(Promedio simple, índice base $2005=100$ )

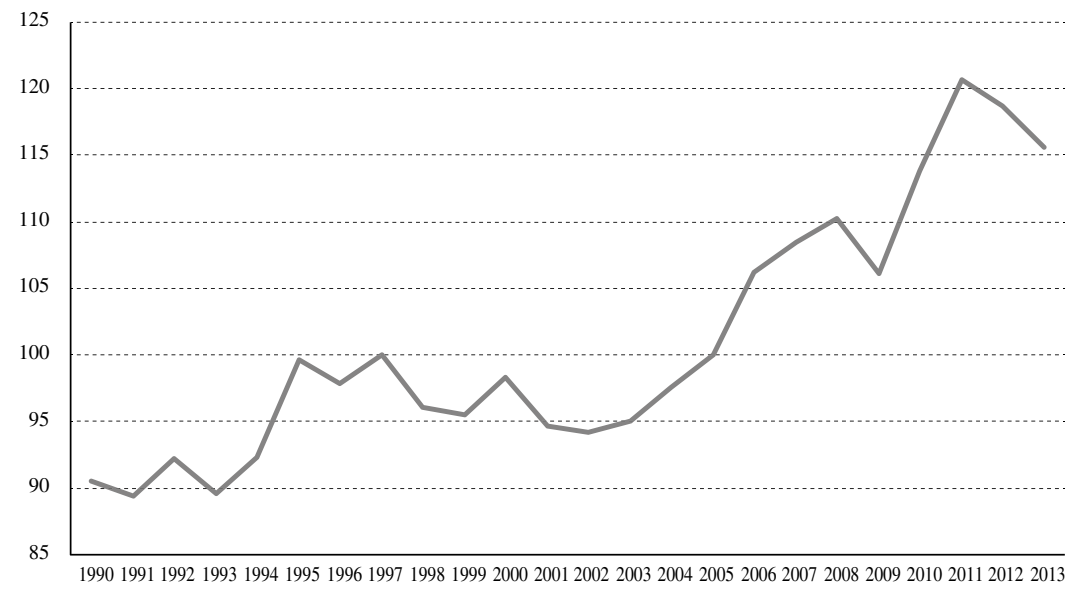

Fuente: elaboración propia sobre la base de datos de CEPALSTAT.

En el gráfico 5 se puede observar cómo en la primera década del siglo XXI el tipo de cambio real bilateral estuvo apreciado con relación a la década previa en el promedio simple de los países de América Latina, en ello influyó, al igual que en el caso de la tasa real de interés que se presenta a continuación, la afluencia de capitales hacia esta región (que redujeron el tipo de cambio nominal) y el mayor control que mostró la inflación. 
GRÁFICO 5

Tipo de cambio real bilateral de América Latina con China, 1990-2013

(Promedio simple, índice base $2005=100$ )

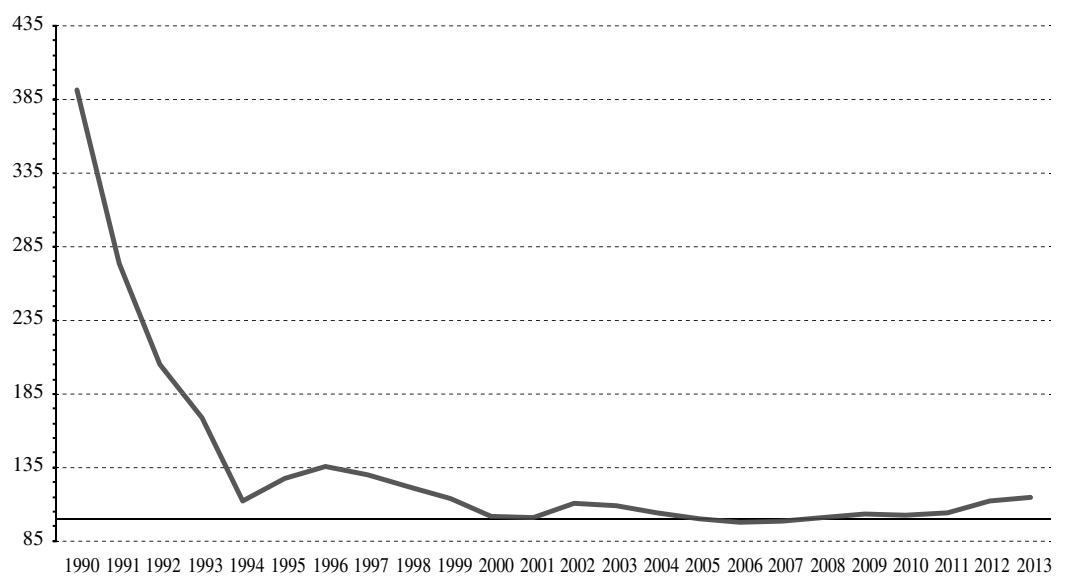

Fuente: elaboración propia sobre la base de datos de cepalstat y del Banco Mundial.

v) " $r$ " corresponde a la tasa real de interés, que ha sido obtenida de la base de datos del Banco Mundial ${ }^{13}$. El gráfico 6 permite observar cómo en el último período (año 2004 en adelante) hubo una disminución significativa de las tasas reales de interés en los países, lo que refleja la mayor liquidez mundial y regional, que a su vez se vio contenida por tasas de inflación significativamente reducidas con respecto al promedio de la década de 1990.

13 Con la excepción del Ecuador y El Salvador, en cuyo caso se utilizaron datos de CEPALSTAT dado que las series del Banco Mundial estaban incompletas para esos países.

GRÁFICO 6

América Latina: tasa de interés real, 1990-2013

(Promedio simple)

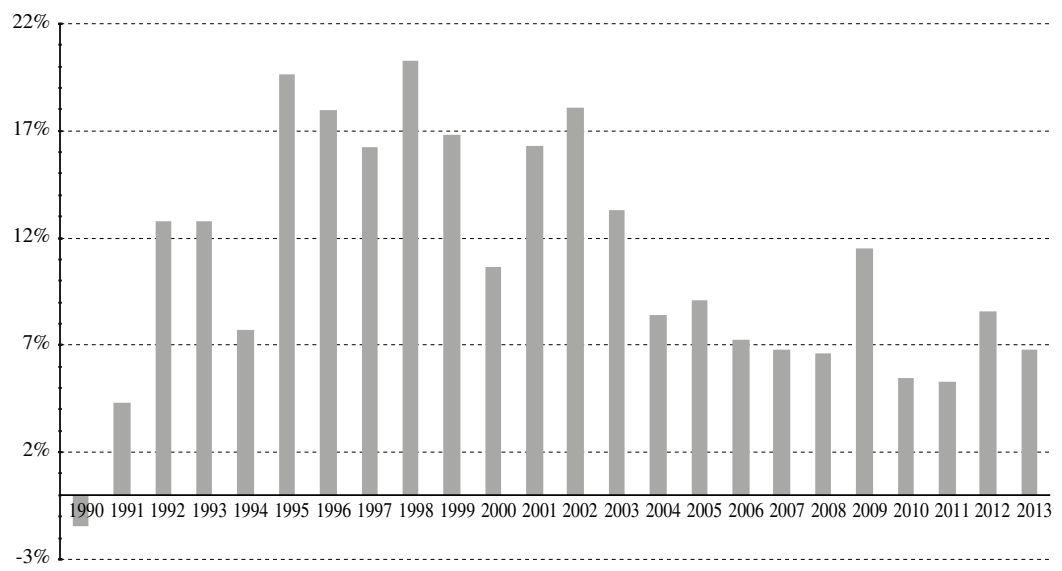

Fuente: elaboración propia sobre la base de datos del Banco Mundial. 
a ese destino, por la vía de un mayor (menor) incentivo a exportar debido a la mayor (menor) ganancia y poder de compra de las exportaciones.

- Un aumento (disminución) exógeno en el tipo de cambio real promueve - ceteris paribusun incremento (merma) en las exportaciones de América Latina a ese destino, debido al incentivo de una mejora (empeoramiento) en la competitividad de los productos exportados hacia China.

- Un aumento (disminución) exógeno en la tasa real de interés provoca - ceteris paribus - una disminución (incremento) en las cantidades exportadas a China, que se fundamenta en el mayor (menor) costo de oportunidad.

En las estimaciones, y dado que el objetivo consistió en estimar elasticidades, todas las variables, excepto la tasa real de interés (que tiene algunos valores negativos), han sido expresadas en sus respectivos logaritmos.

\section{Estrategia de estimación}

La estrategia de estimación elegida para modelar la ecuación (1) consistió en la utilización de una metodología que permitiera considerar el efecto dinámico de las exportaciones a China. La importancia de utilizar esta clase de metodologías es que posibilita obtener las elasticidades de largo plazo que tienen en cuenta el efecto autorregresivo derivado de la presencia de cierta continuidad en los mercados de exportación entre América Latina y China: una vez que se establecen los compromisos entre las partes y se comienza a exportar, generalmente dichos compromisos se mantienen en vigencia durante varios años. Conforme a lo anterior, se determinó la siguiente especificación:

$x_{i, t}=\alpha_{1} x_{i, t-1}+\alpha_{2} y_{t}+\alpha_{3} t_{t o t}+\alpha_{4} t c r_{i, t}+\alpha_{5} r_{i, t}+\mu_{i, t}$

Para abordar esta clase de modelos, los estimadores de mínimos cuadrados ordinarios MCO), efectos fijos y efectos aleatorios resultan sesgados e inconsistentes (Hsiao, 1986; Baltagi, 1995), por ello se recurre al estimador de Arellano y Bond (1991) en el que se utilizan los rezagos de la variable dependiente como instrumentos para su estimación.

El estimador de Arellano y Bond emplea el método generalizado de momentos (MGM) para la estimación de un modelo dinámico de la forma:

con

$$
y_{i, t}=\alpha y_{i, t-1}+\beta x_{i, t}+\mu_{i t}
$$

donde " $y_{i, t}$ " es la variable de interés a explicar por su propio rezago y por otras variables exógenas de interés " $\chi_{i, t}$ ". Asimismo, el término de error $\mu_{i t}$ está compuesto de dos partes: una con un componente inobservable de cada unidad cross section (efectos individuales) $\eta_{i}$, y de un error idiosincrático de ruido blanco $\varepsilon_{i, t}$. Se utiliza para paneles largos con períodos temporales reducidos, y requiere que no exista autocorrelación en el error idiosincrático. El estimador se construye con las primeras diferencias a fin de remover los efectos de nivel en paneles, usando instrumentos para formar las condiciones de los diferentes momentos. Las condiciones de los momentos son formadas por las primeras diferencias del error y los demás instrumentos (variables dependientes rezagadas, entre otras).

El requerimiento de esta clase de estimadores con respecto a la dimensión del panel (relativamente elevados $i$ respecto a $t$ ), tiene vinculación directa con la propuesta del autor del presente artículo de obtener aquellas elasticidades que reflejen con mayor certidumbre el período de auge reciente. Por ello, a pesar de contar con datos desde el año 1990, las estimaciones bajo esta metodología se realizaron para el período muestral 2003-2013 ${ }^{14}$.

En las estimaciones realizadas se consideraron $a$ priori todas las variables explicativas descritas en el modelo (1), aunque el procedimiento de estimación consistió en ir descartando aquellas variables que no evidenciaban la significatividad estadística a niveles usuales. Por lo tanto, en los resultados que se presentan a continuación solo se muestran aquellas variables que han dejado ver un buen comportamiento estadístico.

\section{a) Estimación con exportaciones generales}

Mediante el procedimiento descrito en la subsección anterior, se procedió a estimar el modelo (2). Los resultados de la estimación en que se considera como variable dependiente al logaritmo del total de las exportaciones de cada país latinoamericano a China en términos reales, se presentan en el cuadro 2, donde puede apreciarse que el componente autorregresivo tiene una muy elevada significatividad estadística y una memoria de 0,47 unidades por cada unidad exportada en el período previo. Asumiendo este componente autorregresivo, la elasticidad ingreso de corto plazo de las exportaciones

\footnotetext{
${ }^{14}$ La elección de comenzar el período muestral de las estimaciones en el año 2003 se basó en dos criterios complementarios: el importante dinamismo que se generó sobre todo a partir de dicho año (véase Rosales y Kuwayama, 2012), y la salida de la relevante crisis económica que afectó a buena parte de los países de la región latinoamericana en 2002.
} 
asciende a 0,79 puntos porcentuales por cada punto porcentual de variación en el PIB de China, mientras que la elasticidad de largo plazo ${ }^{15}$ alcanza a 1,51 ; en otras palabras, por cada $1 \%$ de crecimiento adicional del PIB chino, las exportaciones latinoamericanas crecerían, en promedio, $1,51 \%$.

CUADRO 2

\section{Estimación del modelo general}

\begin{tabular}{lcc}
\hline & \multicolumn{2}{c}{ (Variable dependiente: lexpo_reales) } \\
\cline { 2 - 3 } & Parámetro & Estadístico - Z \\
\hline lexpo_reales (-1) & 0,47 & $7,55 * * *$ \\
lgdp_ch & 0,79 & $4,33 * * *$ \\
ltot & - & - \\
ltcr & - & - \\
$\mathrm{r}$ & - & \\
$\mathrm{N}^{\text {o de observaciones }}$ & \multicolumn{2}{c}{187} \\
$\mathrm{~N}^{\text {o de países }}$ & \multicolumn{3}{c}{17} \\
Período de estimación & \multicolumn{2}{c}{$2003-2013$} \\
\hline
\end{tabular}

Fuente: elaboración propia.

*** Significancia estadística al $1 \%$.

lexpo_reales: logaritmo de las exportaciones.

lgdp_ch: logaritmo del PIB de China.

ltot: logaritmo de los términos de intercambio.

ltcr: logaritmo del tipo de cambio real.

$\mathrm{r}$ : tasa real de interés.

A continuación se procedió a evaluar la prueba de Sargan, que chequea restricciones de sobreidentificación bajo la hipótesis nula de que las restricciones son válidas. En otros términos, es una prueba acerca de la validez de las variables instrumentales, que corrobora que estas no posean correlación con los residuos y, por ende, que sean instrumentos válidos. Los resultados de dicha prueba para la estimación anterior se presentan en el cuadro 3, en el que puede observarse que el modelo estimado no rechaza la hipótesis nula de poseer instrumentos válidos.

CUADRO 3

\section{Prueba de Sargan}

\begin{tabular}{lc}
\hline & Valores \\
\hline Chi-Cuadrado & 170 \\
Valor $p$ & 0,50
\end{tabular}

Fuente: elaboración propia.

$\mathrm{H}_{0}$ : restricciones válidas (variables instrumentales válidas).

$\mathrm{H}_{1}$ : sobreidentificación.

15 Que viene dada por: $\frac{\alpha_{2}}{1-\alpha_{1}}$. b) Estimación por tipo de producto exportado

Un interrogante a responder consistió en estudiar las diferentes elasticidades del comercio de América Latina con China en función del tipo de canasta de productos exportados. Para ello, se construyeron las series a valores constantes para los siguientes grandes agregados: i) agricultura, caza, silvicultura y pesca; ii) explotación de minas y canteras, y iii) industria manufacturera, canastas correspondientes a las grandes divisiones de la nomenclatura de la Clasificación Industrial Internacional Uniforme de Todas las Actividades Económicas (CIIU), segunda revisión, obtenidas del Banco de Datos del Comercio Exterior de América Latina y el Caribe (BADECEL) de la CEPAL. Estas series fueron deflactadas utilizando los índices de precios de los productos básicos del sector externo de la CEPAL ${ }^{16}$, quedando estas valoradas a dólares de 2005 .

La selección de los países integrantes en cada serie siguió el cumplimiento conjunto de los siguientes criterios metodológicos: i) el país pertenece a la categoría si la exportación en los rubros a, b y c es significativa en su participación total de exportaciones a China $(>=10 \%)$; y, además, ii) el país pertenece a la categoría si las exportaciones son iguales o superiores al $0,4 \%$ del total de las exportaciones latinoamericanas del sector al país asiático.

En el gráfico 7 puede observarse cómo durante la década de 1990, prácticamente las únicas exportaciones que la región realizaba a China correspondían a bienes de la industria manufacturera (que alcanzaban una participación del $84 \%$ sumando las tres grandes divisiones), lo cual se modificó de manera pronunciada a partir del auge de los productos básicos de los primeros años del siglo XXI, donde empezaron a cobrar gran relevancia las exportaciones de minas y canteras y las agropecuarias, que alcanzaron en conjunto una proporción similar en el comercio a la de la industria manufacturera.

Las estimaciones por grandes divisiones de la ecuación (1) se realizaron nuevamente mediante la metodología de Arellano y Bond (1991). En el cuadro 4 se presentan los resultados alcanzados, donde se destaca que la mayor elasticidad de largo plazo corresponde a los productos agropecuarios $(1,60)$, seguida por la de los productos de minas y canteras $(1,43)$, y finalmente aparecen con elasticidad ingreso menor a la unitaria los productos

16 Los índices de precios utilizados fueron los siguientes: 1) productos agropecuarios, 2) minerales, y, 3) energía, los que se utilizaron respectivamente para deflactar las series i) agricultura, caza, silvicultura y pesca, ii) explotación de minas y canteras, y iii) industria manufacturera. 
GRÁFICO 7

Exportaciones de América Latina a China por grandes divisiones, 1990-2011 (En millones de dólares a precios de 2005)

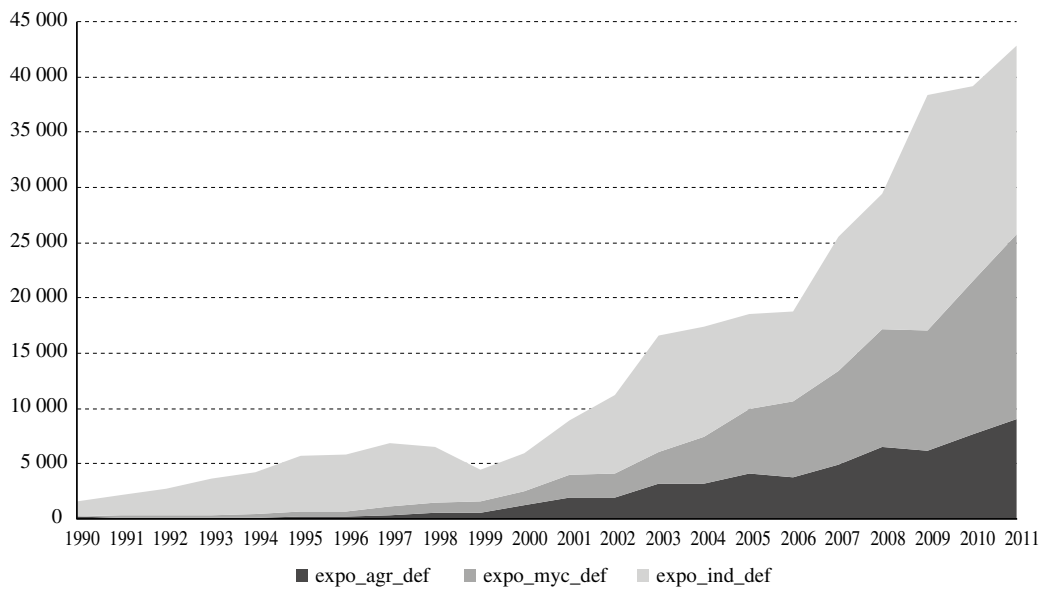

Fuente: elaboración propia sobre la base de información del Banco de Datos del Comercio Exterior de América Latina y el Caribe (BADECEL). expo agr_def: agricultura, caza, silvicultura y pesca; expo_myc_def: explotación de minas y canteras; expo_ind_def: industria manufacturera.

\section{Estimación de modelos por tipo de productos exportados}

\begin{tabular}{|c|c|c|c|c|c|c|}
\hline & \multicolumn{2}{|c|}{$\begin{array}{l}\text { Variable dependiente: } \\
\text { lexpo_agr_def }\end{array}$} & \multicolumn{2}{|c|}{$\begin{array}{l}\text { Variable dependiente: } \\
\text { lexpo_myc_def }\end{array}$} & \multicolumn{2}{|c|}{$\begin{array}{l}\text { Variable dependiente: } \\
\text { lexpo_ind_def }\end{array}$} \\
\hline & Parámetro & Estadístico - Z & Parámetro & Estadístico - Z & Parámetro & Estadístico - Z \\
\hline lexpo_x_def (-1) & 0,41 & $3,74 * * *$ & 0,47 & $4,69 * * *$ & 0,30 & $2,74 * * *$ \\
\hline lgdp_ch & 0,95 & $3,23 * * *$ & 0,75 & $1,97 * *$ & 0,57 & $2,05 * *$ \\
\hline ltot & - & - & - & - & - & - \\
\hline ltcr & - & - & & & - & - \\
\hline $\mathrm{r}$ & - & - & $-3,74$ & $-2,80 * * *$ & - & - \\
\hline $\mathrm{N}^{\mathrm{o}}$ de observaciones & \multicolumn{2}{|c|}{44} & \multicolumn{2}{|c|}{53} & \multicolumn{2}{|c|}{72} \\
\hline $\mathrm{N}^{\mathrm{o}}$ de países & \multirow{2}{*}{\multicolumn{2}{|c|}{5}} & \multicolumn{2}{|c|}{7} & \multirow{2}{*}{\multicolumn{2}{|c|}{8}} \\
\hline Período de estimación & & & \multicolumn{2}{|c|}{ 2003-2011 } & & \\
\hline
\end{tabular}

Fuente: elaboración propia.

** Significancia estadística al 5\%.

*** Significancia estadística al $1 \%$.

lexpo_x_def: logaritmo exportaciones.

lexpo_agr_def: logaritmo exportaciones agricultura, caza, silvicultura y pesca.

lexpo_myc_def: logaritmo explotación de minas y canteras.

lexpo_ind_def: logaritmo exportaciones industria manufacturera.

lgdp_ch: logaritmo del PIB de China.

ltot: logaritmo de los términos de intercambio.

ltcr: logaritmo del tipo de cambio real.

$r$ : tasa real de interés. 
industriales $(0,82)$. Las elasticidades de corto plazo siguen las mismas características que sus contrapartes de largo plazo, siendo de 0,$95 ; 0,75 ;$ y 0,57 , respectivamente.

El resultado de las estimaciones reviste una relación directa con lo que se podría esperar ex ante, en términos del grado de desarrollo alcanzado por la economía China durante el período analizado, y de las necesidades derivadas de ese grado que concuerdan con una mayor elasticidad ingreso por productos primarios y minerales, los que son utilizados para acompañar la construcción de las grandes ciudades y proveer de insumos a la propia producción agropecuaria del país asiático, posibilitando alimentar a la creciente población que migra del sector rural a las nuevas urbes. Por su parte, las manufacturas latinoamericanas, que en las estimaciones registran una menor elasticidad ingreso, dan cuenta de la importancia y el grado de desarrollo que tiene este sector productivo

\section{V}

\section{Conclusiones}

Durante la última década (particularmente desde el año 2003), el comercio de América Latina con China se ha tornado estratégico para varios países de la región. Además de los beneficios directos derivados del intercambio comercial, los ingresos provenientes de los gravámenes sobre las exportaciones latinoamericanas han conformado un activo importante de las arcas fiscales y de la acumulación de reservas internacionales.

En la primera sección del documento se destacó la importancia estratégica que tiene China para América Latina como receptora de buena parte de las exportaciones regionales ${ }^{17}$. Por ello, en la actualidad existe una cierta preocupación respecto del impacto que tendrá en la región el desempeño de la economía asiática en el mediano plazo, en circunstancias que varios expertos advierten acerca de una desaceleración de las tasas de crecimiento que se materializaron en las últimas dos décadas (Chivakul, 2014).

Para dar respuesta al posible impacto que esta desaceleración podría tener en América Latina, en el presente trabajo se estimó la elasticidad ingreso de largo plazo de las exportaciones regionales al mercado

17 En este trabajo no se ha analizado la importancia geopolítica de China para la región, o de otras esferas de influencia económica (como la inversión extranjera directa (IED) de ese país en América Latina). en China, y del papel sustitutivo de los productos latinoamericanos en estos intercambios.

$\mathrm{Al}$ igual que en la estimación anterior, nuevamente se realizó la prueba de Sargan para chequear la sobreidentificación de las variables utilizadas. Los resultados de la prueba, que pueden verse en el cuadro 5, muestran el no rechazo de la hipótesis nula de instrumentos válidos para los tres modelos estimados.

CUADRO 5

Prueba de Sargan

\begin{tabular}{lccc}
\hline Valores & Modelo 1 & Modelo 2 & Modelo 3 \\
\hline Chi-Cuadrado & 40 & 61 & 72 \\
Valor $p$ & 0,58 & 0,14 & 0,43 \\
\hline
\end{tabular}

Fuente: elaboración propia.

$\mathrm{H}_{0}$ : restricciones válidas (variables instrumentales válidas). $\mathrm{H}_{1}$ : sobreidentificación.

chino. Con ese fin se utilizaron modelos de datos de panel dinámicos siguiendo la metodología propuesta por Arellano y Bond (1991).

Los resultados que arrojaron las estimaciones dan cuenta de elasticidades de largo plazo superiores a la unidad (con la excepción de los productos industriales), aunque con valores moderados. Asimismo, las estimaciones se muestran robustas en cuanto a la selección de las variables utilizadas, lo que fue estudiado mediante la prueba de Sargan.

Al comparar los resultados obtenidos con otros trabajos de la literatura en que se estimaron elasticidades ingreso del comercio de América Latina con China, se han realizado los siguientes avances: la inclusión de una mayor cantidad de países representativos de la región (con relación a Nomura, 2013); la especificación de un modelo econométrico con datos de panel de carácter dinámico (con respecto al trabajo de CEPAL, 2012).

Mirando hacia delante, en las estimaciones del Fondo Monetario Internacional sobre la economía china para los próximos seis años (2014-2019) se proyecta un crecimiento promedio anual del $6,8 \%$. Si este crecimiento se confirmara, las estimaciones arrojadas por el modelo general señalarían que las exportaciones de la región a dicho destino registrarían un crecimiento en torno del $10 \%$ promedio anual. Por su parte, un escenario más conservador, en que se contemple un 
crecimiento promedio anual de la economía china del 4,5\% para igual período, arrojaría un crecimiento de las exportaciones de América Latina al país asiático cercanas al $7 \%$ anual.

La dinámica esperable del comercio bilateral implicará que continuarán los desafíos en materia de infraestructura y logística que deberán atender los gobiernos. Al respecto, en algunos documentos (CEPAL,
2010; Perrotti y Sánchez, 2011; Sánchez y Perrotti, 2012) ya se ha alertado sobre la problemática de la estrechez de la infraestructura y su negativa repercusión en el comercio. Resulta perentorio, por lo tanto, que se realicen las inversiones en infraestructura necesarias para evitar potenciales cuellos de botella que surjan del comercio exterior en general, y del intercambio con China en particular.

\section{Bibliografía}

Anderson, J. (2010), "The gravity model", NBER Working Paper Series, $\mathrm{N}^{\circ} 16576$, Cambridge, Massachusetts, National Bureau of Economic Research.

Arellano, M. y S. Bond (1991), "Some tests of specification for panel data: Monte Carlo evidence and an application to employment equations", Review of Economic Studies, vol. 58, № 2, Wiley Blackwell.

Baltagi, B. (1995), Econometric Analysis of Panel Data, Wiley.

Banco Mundial (2014), "World Development Indicators Database" [en línea] http://data.worldbank.org/.

CEPAL (Comisión Económica para América Latina y el Caribe) (2012), Panorama de la Inserción Internacional de América Latina y el Caribe, 2011-2012 (LC/G.2547-P), Santiago de Chile. Publicación de las Naciones Unidas, $\mathrm{N}^{\circ}$ de venta: S.12.II.G.5.

(2010), La hora de la igualdad: Brechas por cerrar, caminos por abrir (LC/G.2432(SES.33/3)), Santiago de Chile.

(s/f), "Cepalstat. Base de Datos y Publicaciones Estadísticas" [en línea] http://estadisticas.cepal.org

Chivakul, M. (2014), "Economic health check: China would benefit from slower but safer growth", Fondo Monetario Internacional (FMI), julio [en línea] http://www.imf.org/external/pubs/ft/ survey/so/2014/CAR073014A.htm.

comtrade (Base de Datos Estadísticos sobre el Comercio de Mercaderías) [en línea] http://comtrade.un.org/data/.

De Benedictis, L. y D. Taglioni (2011), "The gravity model in international trade" [en línea] http://works.bepress.com/cgi/ viewcontent.cgi?article $=1019 \&$ context=luca_de_benedictis.

FMI (Fondo Monetario Internacional) (2014), "The World Economic Outlook Databases" [en línea] http://www.imf.org/external/ns/ cs.aspx?id=28.

Hausman, J.A. (1978), "Specification tests in econometrics", Econometrica, vol. 46, $\mathrm{N}^{\circ}$ 6, Nueva York, The Econometric Society.

Hsiao, C. (1986), Analysis of Panel Data, Cambridge, Cambridge University Press.

Jenkins, R. (2011), “El 'efecto China' en los precios de los productos básicos y en el valor de las exportaciones de América Latina”, Revista de la CEPAL, $\mathrm{N}^{\circ} 103$ (LC/G.2487-P), Santiago de Chile, abril.

Kao, C. (1999), "Spurious regression and residual-based tests for cointegration in panel data", Journal of Econometrics, vol. $90, \mathrm{~N}^{\circ} 1$, Amsterdam, Elsevier.

Mulder, N. (2006), "Aprovechar el auge exportador de productos básicos evitando la enfermedad holandesa", serie Comercio Internacional, $\mathrm{N}^{\circ} 80$ (LC/L.2627-P), Santiago de Chile, Comisión Económica para América Latina y el Caribe (CEPAL). Publicación de las Naciones Unidas, $\mathrm{N}^{\circ}$ de venta: S.06.II.G.151.

Nomura (2013), Global Markets Research. Anchor Report, julio.

Perrotti D. y R. Sánchez (2011), "La brecha de infraestructura en América Latina y el Caribe", serie Recursos Naturales e Infraestructura, $\mathrm{N}^{\circ} 153$ (LC/L.3342), Santiago de Chile, Comisión Económica para América Latina y el Caribe (CEPAL).

Rosales, O. y M. Kuwayama (2012), "China y América Latina y el Caribe: Hacia una relación económica y comercial estratégica", serie Libros de la CEPAL, $\mathrm{N}^{\circ} 114$ (LC/G.2519-P), Santiago de Chile, Comisión Económica para América Latina y el Caribe (CEPAL). Publicación de las Naciones Unidas, $\mathrm{N}^{\circ}$ de venta: S.12.II.G.12.

(2007), "América Latina al encuentro de China e India: Perspectivas y desafíos en comercio e inversión", Revista de la CEPAL, $\mathrm{N}^{\circ} 93$ (LC/G.2347-P), Santiago de Chile, diciembre.

Sánchez, R. y D. Perrotti (2012), "Looking into the future: big full containerships and their arrival to South American Ports", Maritime Policy and Management, vol. 39, $\mathrm{N}^{\circ} 6$, Taylor \& Francis.

SELA (Sistema Económico Latinoamericano y del Caribe) (2010), Evolución reciente de las relaciones económicas entre la República Popular China y América Latina y el Caribe. Mecanismos institucionales y de cooperación para su fortalecimiento (SP/ CL/XXXVI.O/Di No 12-10), Caracas, octubre.

Wooldridge, J. (2010), Econometric Analysis of Cross Section and Panel Data, Cambridge, Massachusetts, The MIT Press. 